
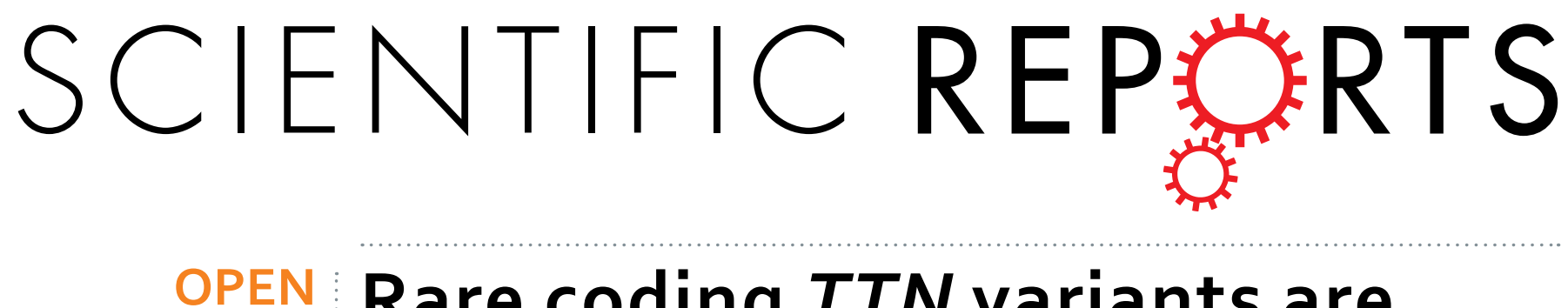

\title{
Rare coding TTN variants are associated with electrocardiographic QT interval in the general
}

Received: 30 March 2016

Accepted: 01 June 2016

Published: 20 June 2016 population

\author{
Ashish Kapoor ${ }^{1,}{ }^{,}, K^{2}$ iranmayee Bakshy ${ }^{1,}{ }^{,}$, Linda Xu$^{1}$, Priyanka Nandakumar ${ }^{1}$, Dongwon Lee ${ }^{1}$, \\ Eric Boerwinkle ${ }^{2}$, Megan L. Grove ${ }^{2}$, Dan E. Arking ${ }^{1}$ \& Aravinda Chakravarti ${ }^{1}$
}

We have shown previously that noncoding variants mapping around a specific set of 170 genes encoding cardiomyocyte intercalated disc (ID) proteins are more enriched for associations with QT interval than observed for genome-wide comparisons. At a false discovery rate (FDR) of $5 \%$, we had identified 28 such ID protein-encoding genes. Here, we assessed whether coding variants at these 28 genes affect $Q T$ interval in the general population as well. We used exome sequencing in 4,469 European American (EA) and 1,880 African American (AA) ancestry individuals from the population-based ARIC (Atherosclerosis Risk In Communities) Study cohort to focus on rare (allele frequency $<1 \%$ ) potentially deleterious (nonsynonymous, stop-gain, splice) variants ( $n=2,398$ for $E A ; n=1,693$ for $A A$ ) and tested their effects on standardized QT interval residuals. We identified 27 nonsynonymous variants associated with QT interval (FDR 5\%), 22 of which were in TTN. Taken together with the mapping of a OT interval GWAS locus near TTN, our observation of rare deleterious coding variants in TTN associated with QT interval show that TTN plays a role in regulation of cardiac electrical conductance and coupling, and is a risk factor for cardiac arrhythmias and sudden cardiac death.

Over the last decade genome wide association studies (GWAS) have been successful in identifying common variants (minor allele frequency (MAF) $\geq 1 \%$ ), mostly non-coding, that underlie genetic variation of common diseases and quantitative traits ${ }^{1}$. Whole exome sequencing- (WES) and/or whole genome sequencing-(WGS) based approaches have extended such screens to include rare coding and non-coding variants $(\mathrm{MAF}<1 \%)^{2}$. However, given the stringent thresholds set for attaining genome-wide significance (after correcting for multiple tests) ${ }^{3}$ and small allelic effects of individual variants ${ }^{4}$, the discovery of new associations has been challenging. With the number of tests being performed running in millions, correcting for the false positive rates results in a high false-negative rate due to which many true associations remain hidden. One way to increase the power to detect these true associations is to perform hypothesis-driven tests, where the search is limited to candidate genes/loci based on ancillary knowledge of genetic mapping, co-expression, protein-protein interaction, subcellular protein localization and others.

The QT interval (MIM 610141), a measurement of the duration of cardiac repolarization on an electrocardiogram (ECG), is a quantitative trait with $\sim 30 \%$ heritability and considerable medical relevance as prolongation and shortening of the QT interval are associated with increased risk of cardiovascular morbidity and mortalit $\mathrm{y}^{5,6}$. Abnormal QT intervals observed in Mendelian disorders, known as long-QT syndrome and short-QT syndrome, are associated with increased risk of cardiac arrhythmias and sudden cardiac death (SCD), and are usually due to rare, high penetrance coding mutations in genes encoding ion channels and their associated proteins ${ }^{7}$. GWAS of the QT interval have identified at least 35 loci influencing trait variation in subjects of European American (EA) ancestry that collectively explain $\sim 8 \%$ of the phenotypic variance in the general population ${ }^{8}$. Among these GWAS loci, a locus on chromosome $1 \mathrm{q}$ containing the gene NOS1AP is the major genetic regulator of QT interval and accounts for $\sim 1 \%$ of the population trait variation ${ }^{9-11}$.

${ }^{1}$ McKusick-Nathans Institute of Genetic Medicine, Johns Hopkins University School of Medicine, Baltimore, Maryland, 21205, USA. 'Division of Epidemiology, Human Genetics and Environmental Sciences, University of Texas Health Science Center, Houston, Texas, 77030, USA. *These authors contributed equally to this work. Correspondence and requests for materials should be addressed to A.C. (email: aravinda@jhmi.edu) 
Recently, we had shown that a) an enhancer variant affecting NOS1AP expression is the functional basis of the observed trait association, b) over-expression of NOS1AP in cardiomyocytes leads to altered cellular electrophysiology, and c) NOS1AP is localized to cardiomyocyte intercalated discs (ID), leading us to propose that NOS1AP regulates QT interval by affecting cardiac electrical conductance and coupling ${ }^{12}$. Based on the localization of NOS1AP to ID we had hypothesized that ID plays a major role in regulating of QT interval and genetic variation in its components underlie the risk of cardiac arrhythmias and SCD. In support of our hypothesis, we showed that compared to genome-wide markers, common variants mapping near a specific set of 170 genes encoding ID proteins (henceforth referred to as ID genes) are significantly enriched for association with QT interval. Specifically, we identified 28 ID genes/loci, including NOS1AP, that showed common variants associations with QT interval (false discovery rate (FDR) 5\%), many of which were missed in the GWAS and were identified by restricting to variants mapping near a functionally enriched set of ID genes ${ }^{12}$. In this paper, we assessed whether rare coding variants in these 28 ID genes are associated with QT interval variation as well. As opposed to GWAS that map common polymorphisms and identify positional markers and loci instead of genes, assessing association with coding variants couples the identification of functional variants with specific genes. Using variants identified in the 28 candidate ID genes by WES in 4,469 EA and 1,880 African American (AA) ancestry individuals from the $\mathrm{ARIC}^{13}$ (Atherosclerosis Risk in Communities) cohort, we identified multiple rare nonsynonymous variants in TTN associated with QT interval variation. Using TTN-specific functional information to annotate coding variants we observed enrichment in association signal and propose that individual protein-specific functional information will be critical in identifying disease/traits variants among a large number of variants identified by sequencing studies.

\section{Subjects and Methods}

Subjects. Of the 15,792 subjects in the ARIC study, we studied 5,718 EA and 2,836 AA ancestry subjects in whom we had access to GWAS ${ }^{8}$ and WES (unpublished) data. The ARIC study subjects and the procedures have been previously described in ${ }^{13}$. Briefly, ARIC is a population-based prospective cohort study of cardiovascular disease in 15,792 subjects aged 45-64 years at baseline (1987-89) who were randomly sampled from four U.S. communities $(\sim 4,000$ per community). Cohort members completed four extensive clinic examinations, conducted every three years between 1987 and 1998, which included medical, social and demographic data. For assessment of QT interval at baseline, 12-lead ECG digital recording and a 2-minute paper recording of a threelead (leads $\mathrm{V}_{1}$, II and $\mathrm{V}_{5}$ ) rhythm strip were made. The QT interval was determined by identifying Q-wave onset and T-wave offset in all three leads. T-wave offset was defined as the point of maximum change of slope as the $\mathrm{T}$-wave merges with the baseline $\mathrm{S}^{5,14}$. All individuals studied and all analyses on their samples were performed according to the Helsinki declarations, were approved by local ethics and institutional review committees of the CHARGE (Cohorts for Heart and Aging Research in Genomic Epidemiology) consortium ${ }^{15}$ and all participants provided informed consent.

Library Preparation, Exome Sequencing, Variant and Genotype Calling. All the steps of library preparation, capture method, sequencing, variant and genotype calling have been detailed elsewhere ${ }^{16}$. All variants analyzed in this study have been submitted to Database of Single Nucleotide Polymorphisms (dbSNP) ${ }^{17}$, Bethesda (MD): National Center for Biotechnology Information, National Library of Medicine under the dbSNP accession: ss1998364261-ss1998377886 (dbSNP Build ID:149).

Variant-level and Sample-level QC. WES was performed on samples from multiple cohorts as part of the CHARGE consortium ${ }^{15}$ and will be described elsewhere (unpublished data). In this study we analyzed only single nucleotide variants (SNVs) and ignored the small insertions/deletions (indels). Variant-level QC applied on the entire sequencing data removed variants with $>20 \%$ missing data, more than 2 observed alleles, monomorphic, or with mean depth of greater than 500 -fold. Then, separately within each ancestry group, variants that deviated from Hardy-Weinberg equilibrium $\left(\mathrm{P}<5 \times 10^{-6}\right)$ were filtered out. Next, within each cohort, samples with $>$ $20 \%$ missing data were removed. We, then, assessed sequencing data from 5,718 EA and 2,836 AA ARIC subjects. In addition, we filtered out 1,249 EA and 956 AA ancestry ARIC subjects based on genetic relatedness (based on common variant genotypes ${ }^{8}$ ), genetic outliers, missing phenotype (no raw QT interval measurement), history of cardiac disease, abnormal ECG findings, or those on known QT-altering medications ${ }^{13}$, leaving 4,469 EA and 1,880 AA ancestry subjects for analysis.

Variant Annotation. For each of the 28 candidate ID genes we first identified the most abundant human cardiac RefSeq ${ }^{18}$ transcript using RNA-seq-based gene expression data generated by the UCSD Human Reference Epigenome Mapping Project ${ }^{19}$ (GEO accession: GSE16256). WES variants observed were then annotated with respect to this transcript using ANNOVAR ${ }^{20,21}$. Nonsynonymous variants were further annotated with respect to the 46-species vertebrate base-wise conservation score generated using phyloP ${ }^{22}$ (available from the UCSC Genome Browser ${ }^{23,24}$ ) and overlap with a known protein domain (from the Human Protein Reference Database ${ }^{25,26}$ ). Nonsynonymous variants in $T T N$ were also annotated with respect to the sarcomeric bands ${ }^{27}$. For all variants found to be associated with QT interval, we assessed the predicted functional effect using SIFT ${ }^{28}$ and PolyPhen- $2^{29}$ and also looked up allele counts in the Exome Aggregation Consortium (ExAC) browser ${ }^{30}$.

Statistical Analyses. We used multivariate linear regression to correct the raw QT interval measured in the ECG for known covariates: heart-rate (RR interval on ECG) ${ }^{31}$, age ${ }^{32}$ and $\operatorname{sex}^{33}$ as well as genotypes of common polymorphisms representing 34 QT interval GWAS hits; rs1805128 in KCNE1 was not observed in ARIC subjects $^{8}$. Single variant quantitative trait association analysis was performed as implemented in PLINK $^{34,35}$ using 


\begin{tabular}{|c|c|c|c|c|c|c|c|c|}
\hline Gene & Transcript $^{1}$ & ORF length & Synonymous & Nonsynonymous & Stopgain & Splice & All & $\begin{array}{l}\text { \#Variants/ } \\
\text { ORF length }\end{array}$ \\
\hline ANKRD30A & NM_052997 & 4026 & 42 & 106 & 3 & 3 & 154 & 0.038 \\
\hline ATP1B1 & NM_001677 & 912 & 15 & 3 & 0 & 0 & 18 & 0.020 \\
\hline CAV1 & NM_001172896 & 444 & 4 & 11 & 0 & 0 & 15 & 0.034 \\
\hline CAV2 & NM_001233 & 489 & 3 & 10 & 0 & 0 & 13 & 0.027 \\
\hline CD59 & NM_001127223 & 387 & 9 & 5 & 0 & 0 & 14 & 0.036 \\
\hline $\mathrm{CDH11}$ & NM_001797 & 2391 & 34 & 45 & 0 & 0 & 79 & 0.033 \\
\hline $\mathrm{CDH} 2$ & NM_001792 & 2721 & 32 & 42 & 0 & 0 & 74 & 0.027 \\
\hline CIB1 & NM_001277764 & 696 & 10 & 11 & 1 & 0 & 22 & 0.032 \\
\hline ERBB4 & NM_005235 & 3927 & 35 & 53 & 1 & 1 & 90 & 0.023 \\
\hline KCNJ4 & NM_152868 & 1338 & 27 & 8 & 0 & 0 & 35 & 0.026 \\
\hline KCNK3 & NM_002246 & 1185 & 12 & 8 & 0 & 0 & 20 & 0.017 \\
\hline LRFN2 & NM_020737 & 2370 & 46 & 32 & 0 & 0 & 78 & 0.033 \\
\hline NOS1AP & NM_014697 & 1521 & 24 & 18 & 0 & 0 & 42 & 0.028 \\
\hline NRAP & NM_001261463 & 5196 & 76 & 171 & 7 & 8 & 262 & 0.050 \\
\hline PARVA & NM_018222 & 1239 & 21 & 21 & 0 & 2 & 44 & 0.036 \\
\hline PKP2 & NM_004572 & 2646 & 25 & 63 & 1 & 2 & 91 & 0.034 \\
\hline PKP4 & NM_003628 & 3579 & 45 & 73 & 1 & 2 & 121 & 0.034 \\
\hline PRKCA & NM_002737 & 2019 & 28 & 22 & 1 & 0 & 51 & 0.025 \\
\hline PTK2 & NM_001199649 & 3198 & 28 & 47 & 1 & 1 & 77 & 0.024 \\
\hline SCN5A & NM_000335 & 6048 & 109 & 130 & 2 & 0 & 241 & 0.040 \\
\hline$S G C Z$ & NM_139167 & 939 & 6 & 32 & 1 & 1 & 40 & 0.043 \\
\hline SIPA1L1 & NM_001284247 & 5412 & 66 & 88 & 0 & 0 & 154 & 0.028 \\
\hline SLC16A1 & NM_003051 & 1503 & 13 & 20 & 0 & 0 & 33 & 0.022 \\
\hline SLC4A1 & NM_000342 & 2736 & 44 & 83 & 0 & 0 & 127 & 0.046 \\
\hline SLC8A1 & NM_021097 & 2922 & 35 & 45 & 0 & 0 & 80 & 0.027 \\
\hline SPTBN1 & NM_003128 & 7095 & 127 & 87 & 0 & 0 & 214 & 0.030 \\
\hline TLN1 & NM_006289 & 7626 & 95 & 95 & 0 & 0 & 190 & 0.025 \\
\hline \multirow[t]{2}{*}{ TTN } & NM_001267550 & 107976 & 1024 & 2448 & 14 & 13 & 3499 & 0.032 \\
\hline & & & 2035 & 3777 & 33 & 33 & 5878 & \\
\hline
\end{tabular}

Table 1. Gene symbol, most abundant cardiac transcript, ORF length, number and type of coding variants observed in EA and AA ARIC subjects, and number of coding variants per coding base for the 28 ID genes. ${ }^{1}$ Most abundant human cardiac transcript.

a linear regression model with standardized QT interval residuals. False discovery rates were calculated from $p$-values using the Benjamini-Hochberg procedure ${ }^{36}$.

\section{Results}

Of the 5,718 and 2,836 self-identified EA and AA ARIC individuals in which WES was performed, 1,249 EA and 956 AA samples, respectively, were excluded for the following reasons a) suspected first-degree relative of an included individual based on genome-wide genotype data, b) genetic outlier based on average identity-by-state using PLINK ${ }^{35}$ and $>8$ standard deviations along any of the first 10 principal components in EIGENSTRAT ${ }^{37}$ after 5 iterations, c) genotypic sex not matching phenotypic sex, d) discordant with previous genotype data, e) individuals with atrial fibrillation, QRS duration $>120 \mathrm{~ms}$, bundle branch block or intraventricular conduction delay, f) individuals with history of heart failure or myocardial infarction, and g) when available, use of electronic pacemaker or QT-altering drugs. In the remaining 4,469 EA and 1,880 AA ARIC samples, a total of 5,878 coding SNVs (3,830 in EA subjects, 2,982 in AA subjects and overlap of 934 variants) were identified at the 28 candidate ID genes. Coding SNVs were annotated with respect to the most abundant human cardiac RefSeq transcript using ANNOVAR. Table 1 lists the 28 candidate genes, corresponding transcripts and ORF lengths, types and numbers of coding SNVs observed combined in the EA and AA ARIC subjects, and number of coding SNVs per coding base (see Supplementary Tables S1 and S2 for EA and AA only variants, respectively). A total of 2,035 synonymous, 3,777 nonsynonymous, 33 stop-gain and 33 canonical splice site SNVs were observed across all 28 ID genes. The number of coding SNVs/coding base varied from 0.017 (KCNK3) to 0.050 (NRAP) with a mean and median of 0.031 , across all 28 ID genes. For each coding SNV we calculated minor allele frequency using the genotypes from the EA and AA ARIC samples separately and as expected, the majority of the coding variants were rare (Fig. 1). Of all synonymous variants, $~ 91 \%(1182 / 1303)$ in EA subjects and $~ 84 \%(935 / 1111)$ in AA subjects had allele frequency $<1 \%$, and of all nonsynonymous, stop-gain and splice variants, $~ 95 \%(2398 / 2527)$ in EA subjects and $\sim 91 \%(1693 / 1871)$ in AA subjects had allele frequency of $<1 \%$. Nearly $60 \%$ of all synonymous variants and $65 \%$ of all nonsynonymous, stop-gain and splice variants were observed as singletons in EA subjects; the corresponding values for AA subjects were $44 \%$ and $52 \%$, respectively (Fig. 1). 
a)

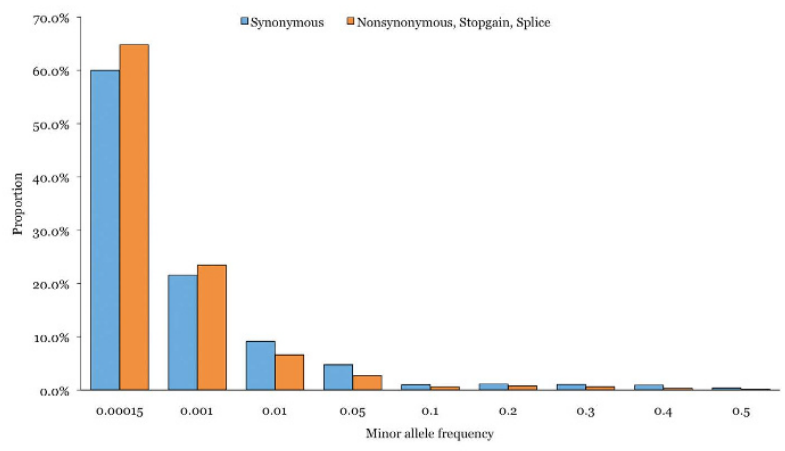

b)

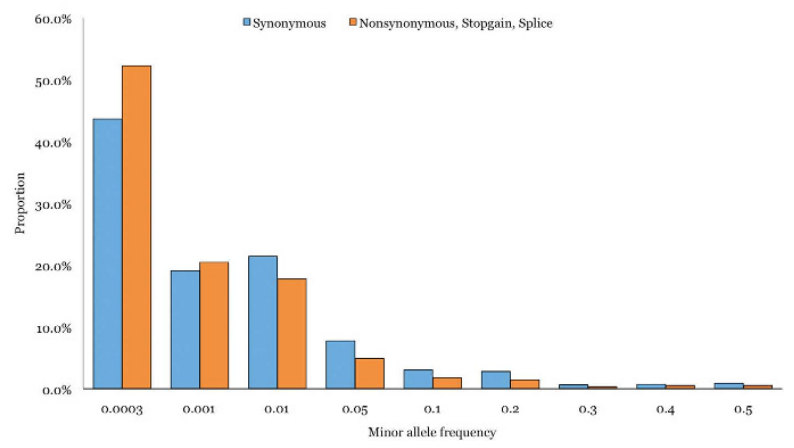

Figure 1. Minor allele frequency distribution of coding variants observed at 28 ID genes in EA (a) and AA (b) ARIC subjects.

\begin{tabular}{|l|c|c|c|c|c|c|c|c|c|}
\hline Chr:Position (hg19) & Gene & cDNA change & $\begin{array}{c}\text { Protein } \\
\text { change }\end{array}$ & MAF & Beta & P & $\begin{array}{c}\text { PhyloP } \\
\text { score }\end{array}$ & $\begin{array}{c}\text { Protein } \\
\text { domain }\end{array}$ & ExAC allele count \\
\hline $2: 179455718$ & $T T N$ & c.G60734A & p.R20245Q & 0.00011 & 8.78 & $1.22 \times 10^{-18}$ & 6.39 & FN2 & $4 / 120604$ \\
\hline $2: 179407497$ & $T T N$ & c.G97084T & p.A32362S & 0.00011 & 5.39 & $6.89 \times 10^{-8}$ & 0.15 & - & - \\
\hline $2: 212522534$ & $E R B B 4$ & c.C1891T & p.H631Y & 0.00022 & 3.69 & $1.73 \times 10^{-7}$ & 4.15 & - & - \\
\hline $2: 179634919$ & $T T N$ & c.A8509T & p.S2837C & 0.00022 & 3.19 & $6.43 \times 10^{-6}$ & 3.73 & IG & $12 / 121362$ \\
\hline $2: 179473995$ & $T T N$ & c.A52042G & p.M17348V & 0.00011 & 4.14 & $3.33 \times 10^{-5}$ & 1.38 & FN3 & $2 / 96388$ \\
\hline $2: 179398282$ & $T T N$ & c.C103060T & p.P34354S & 0.00011 & 4.14 & $3.47 \times 10^{-5}$ & 5.98 & - & - \\
\hline $2: 179629385$ & $T T N$ & c.A9857G & p.K3286R & 0.00011 & 4.11 & $3.81 \times 10^{-5}$ & 5.11 & IGC2 & $15 / 121360$ \\
\hline $2: 179496930$ & $T T N$ & c.C43691G & p.S14564C & 0.00011 & 4.11 & $3.98 \times 10^{-5}$ & 6.35 & - & $5 / 81630$ \\
\hline $2: 179466803$ & $T T N$ & c.C55195T & p.P18399S & 0.00011 & 3.88 & 0.00010 & 4.14 & FN3 & $1 / 120260$ \\
\hline $8: 13959964$ & $S G C Z$ & c.G665T & p.G222V & 0.00011 & 3.78 & 0.00015 & 5.48 & - & - \\
\hline $2: 179424880$ & $T T N$ & c.T85979C & p.I28660T & 0.00011 & 3.77 & 0.00016 & 5.31 & IGC2 & $2 / 120508$ \\
\hline 2:179413763 & $T T N$ & c.G92590A & p.D30864N & 0.00011 & 3.66 & 0.00025 & 3.76 & FN3 & $16 / 120638$ \\
\hline 2:179486250 & $T T N$ & c.A45301C & p.N15101H & 0.00011 & 3.62 & 0.00029 & 3.60 & - & - \\
\hline $2: 179644182$ & $T T N$ & c.A3737T & p.H1246L & 0.00011 & 3.63 & 0.00029 & 3.43 & - & - \\
\hline $3: 38592534$ & $S C N 5 A$ & c.G5326A & p.V1776M & 0.00011 & 3.62 & 0.00029 & 5.80 & - & $3 / 121080$ \\
\hline $2: 179666975$ & $T T N$ & c.G185A & p.R62H & 0.00011 & 3.56 & 0.00037 & 6.21 & IGC2 & $15 / 121364$ \\
\hline
\end{tabular}

Table 2. Coding variants associated with QT interval in EA ARIC subjects.

The raw QT interval measured in the ECG is known to be strongly dependent on heart-rate (RR interval on $\mathrm{ECG})^{31}$, age $\mathrm{e}^{32}$ and $\operatorname{sex}^{33}$ and needs to be corrected for these known covariates before assessing associations. Using a multivariate linear regression model, the raw QT interval measurement in 4,469 EA and 1,880 AA ARIC subjects was corrected for heart-rate, age, sex, and also for the genotypes at the 34 common GWAS variants reported to modulate QT interval in the general population (rs1805128 in KCNE1 was not observed in ARIC subjects) ${ }^{8}$ (see Supplementary Figs S1 and S2 for distribution of corrected QT interval in EA and AA subjects, respectively). Of all coding SNVs identified at the 28 ID genes by WES in EA and AA ARIC subjects, we limited the association analysis to rare $(\mathrm{MAF}<1 \%)$ and potentially deleterious (nonsynonymous, stop-gain, splice) coding variants $(\mathrm{n}=2,398$ for $\mathrm{EA} ; \mathrm{n}=1,693$ for AA). Single variant quantitative trait association analysis using a linear regression model with standardized QT residuals identified 16 and 11 variants, all nonsynonymous, in EA (Table 2) and AA 


\begin{tabular}{|c|c|c|c|c|c|c|c|c|c|}
\hline Chr:Position (hg19) & Gene & cDNA change & $\begin{array}{l}\text { Protein } \\
\text { change }\end{array}$ & MAF & Beta & $\mathbf{P}$ & $\begin{array}{c}\text { PhyloP } \\
\text { score }\end{array}$ & $\begin{array}{l}\text { Protein } \\
\text { domain }\end{array}$ & ExAC allele count \\
\hline 9:35711334 & TLN1 & c.A3937G & p.S1313G & 0.00026 & 15.81 & $3.60 \times 10^{-60}$ & 3.48 & - & $2 / 121374$ \\
\hline 2:179455331 & TTN & c.C61121T & p.P20374L & 0.00053 & 8.85 & $2.26 \times 10^{-37}$ & 6.39 & - & $2 / 119910$ \\
\hline $2: 179447784$ & $T T N$ & c.C65746T & p.R21916W & 0.00026 & 4.98 & $5.87 \times 10^{-07}$ & 0.65 & IGC2 & $13 / 113940$ \\
\hline $3: 38645514$ & SCN5A & c.G1579A & p.G527R & 0.00026 & 4.33 & $1.48 \times 10^{-05}$ & 3.93 & - & $4 / 90200$ \\
\hline 2:179528396 & $T T N$ & c.C36490A & p.P12164T & 0.00026 & 4.10 & $4.03 \times 10^{-05}$ & -0.49 & IG & $7 / 117870$ \\
\hline $2: 179447313$ & $T T N$ & c.C65870T & p.P21957L & 0.00026 & 3.90 & $9.92 \times 10^{-05}$ & 6.22 & FN3 & - \\
\hline 2:179428672-179428673 & $T T N$ & c.82186_82187CA > GT & p.Q27396V & 0.00026 & 3.79 & 0.00015 & $4.74,5.31$ & FN3 & $1 / 120622,1 / 120618$ \\
\hline 2:179451505 & $T T N$ & c.G64123A & p.V21375M & 0.00026 & 3.79 & 0.00015 & 6.37 & FN3 & $1 / 120562$ \\
\hline 2:179497341 & $T T N$ & c.G43392A & p.M14464I & 0.00026 & 3.79 & 0.00015 & 1.68 & IGC2 & $3 / 120562$ \\
\hline $2: 179434555$ & TTN & c.G76304A & p.C25435Y & 0.00026 & 3.62 & 0.00029 & 4.48 & IGC2 & $1 / 120440$ \\
\hline 2:179440480 & TTN & c.T70379G & p.L23460R & 0.00053 & 2.52 & 0.00036 & 5.13 & FN3 & - \\
\hline
\end{tabular}

Table 3. Coding variants associated with QT interval in AA ARIC subjects.

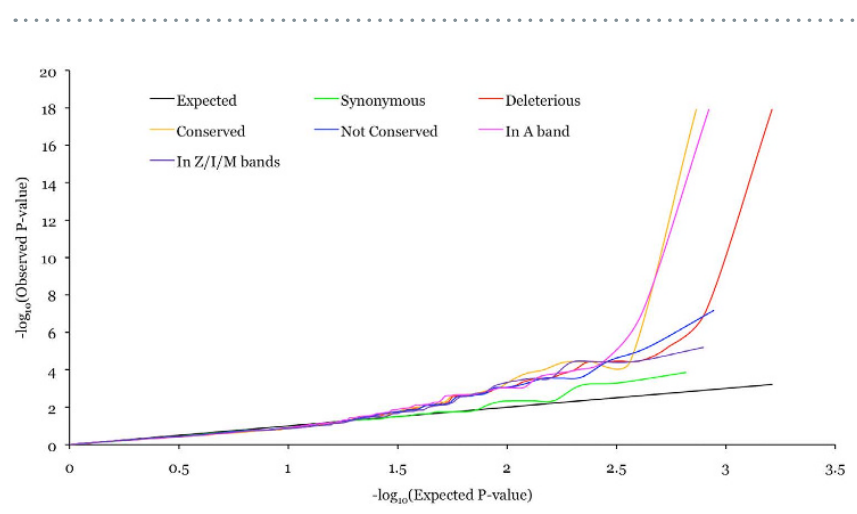

Figure 2. QQ plot for various classes of $T T N$ variants in EA subjects.

(Table 3) subjects, respectively, at a FDR of 5\%. Of the 16 QT interval associated variants in EA subjects 13 were in TTN and 1 each in SCN5A, ERBB4 and SGCZ (Table 2); 14 variants observed as singletons in 10 subjects and 2 variants observed as doubletons in 4 subjects (Supplementary Table S3). Of the 11 QT interval associated variants in AA subjects 9 were in TTN and 1 each in SCN5A and TLN1 (Table 3); 9 variants observed as singletons in 7 subjects and 2 variants observed as doubletons in 4 subjects (Supplementary Table S4). Thus, TTN had 22 out of 27 variants associated with QT interval in EA and AA subjects.

Given that $\sim 80 \%$ of the QT interval associated variants in this study were in TTN we focused on all coding variants in TTN and assessed whether classifying variants into various categories based on function, conservation and sarcomeric domain location, irrespective of allele frequency, might uncover additional associated variants. All TTN coding variants were classified by function into two classes: potentially benign (synonymous; 654/549 in EA/AA) and potentially deleterious (nonsynonymous, stop-gain and splice site; 1624/1231 in EA/AA). All nonsynonymous TTN variants were classified by conservation into two classes: conserved (phyloP score $\geq 4$; $732 / 508$ in EA/AA) and not conserved (phyloP score $<4 ; 877 / 710$ in EA/AA). Titin encoded by TTN is the largest protein in humans and is highly expressed in striated muscle cells, where two titin molecules span each sarcomere in opposing polarity. Within the sarcomere, titin is anchored in the Z-line and spans through the Iand A-bands to the M-line. Mutations in TTN are a leading genetic cause of dilated cardiomyopathies (DCM) and a majority of mutations in patients are observed in the $\mathrm{A}$ band ${ }^{27}$, indicating the functional importance of the corresponding part of the protein in normal physiology. To evaluate the effect of sarcomeric domain location all potentially deleterious TTN variants were classified into two classes: in A band (836/625 in EA/AA) and in Z/I/M bands (788/606 in EA/AA). Quantile-quantile (QQ) plots for various classes of variants showed a trend towards association enrichment for conserved nonsynonymous variants and deleterious variants mapping to the $\mathrm{A}$ band (Figs 2 and 3).

\section{Discussion}

In this study we identified multiple rare nonsynonymous variants in TTN associated with QT interval variation in the general population. Identification of these rare coding $T T N$ variants with large genetic effects within a moderately sized subset of population-based ARIC cohort ${ }^{13}$, represented by 4,469 EA and 1,880 AA ancestry subjects, was made feasible by restricting association analysis of WES variants to coding variants identified in 28 ID genes, for which we had prior evidence of genetic association with QT interval based on common, mostly noncoding polymorphisms ${ }^{12}$. In other words, a functional-hypothesis driven approach was necessary to reduce the search space and increase the power to detect novel associations. Also, by performing association analysis based on coding variants we could directly implicate TTN as a causal gene, in contrast to GWAS that mostly identify positional markers. It is important to point out that only 1 out of the 28 ID genes assessed had convincing evidence for 


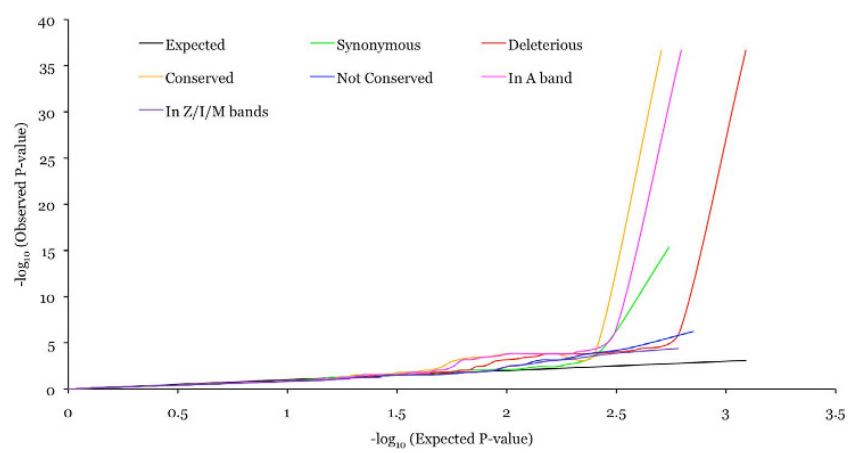

Figure 3. QQ plot for various classes of TTN variants in AA subjects.

coding variants associated with QT interval, indicating that the population level trait variance is largely explained by common noncoding variants.

Titin, encoded by TTN, is an abundant protein of the striated muscle contractile apparatus and plays a key role in sarcomere assembly and functioning of muscle fibers. Beyond the well-documented role played by rare deleterious TTN mutations in causing cardiomyopathies ${ }^{27}$, including arrhythmogenic right ventricular dysplasia ${ }^{38,39}$, and skeletal myopathies, there is growing evidence for the role of deleterious TTN variants in conduction defects, in the presence or absence of cardiomyopathy and sudden cardiac death ${ }^{40,41}$. Our observations of rare coding TTN variants associated with QT interval variation in a population-based cohort further support a broader role of TTN in cardiac physiology. In addition, QT interval GWAS have mapped common noncoding variants near $T T N$ at chromosome $2 q 31.2^{8}$, raising the possibility that TTN expression levels could also modulate cardiac repolarization.

Of the 27 rare nonsynonymous variants associated with QT interval in EA and AA ancestry ARIC subjects, 22 were in TTN, 2 in SCN5A and 1 each in ERBB4, SGCZ and TLN1. Among these 5 genes, based on the number of associated variants uncovered, we conclude that $T T N$ has the potential to regulate QT interval variation. Although, the role of $S C N 5 A$ in regulating QT interval is well established, based on genetic studies of rare Mendelian long-QT syndrome ${ }^{7}$, we observed only 2 variants in SCN5A associated with QT interval variation in the general population. Further association studies would be necessary to assess whether variation in the remaining 3 genes, ERBB4, SGCZ and TLN1, regulates QT interval in the general population. A major limitation of our study is that the 27 QT interval associated variants identified were observed as singletons (23 variants) or doubletons ( 4 variants) (see Supplementary Table S5 for allele counts in different populations), which raises the possibility that some of these could be false-positives. Therefore, performing similar association studies in independent and larger cohorts is, as always, necessary. Another important question that remains to be explored is whether and how these variants affect gene function (see Supplementary Table S6 for predicted functional effect from SIFT ${ }^{28}$ and PolyPhen- $2^{29}$ ). In summary, using a hypothesis-based approach to limit association analysis of WES variants to coding variants in 28 ID genes, we identified multiple rare nonsynonymous TTN variants associated with QT interval variation in the general population, providing evidence of a new role for TTN in cardiac electrical conduction and coupling.

\section{References}

1. Hirschhorn, J. N. Genomewide association studies-illuminating biologic pathways. N. Engl. J. Med. 360, 1699-1701 (2009)

2. Cirulli, E. T. \& Goldstein, D. B. Uncovering the roles of rare variants in common disease through whole-genome sequencing. Nat. Rev. Genet. 11, 415-425 (2010).

3. Risch, N. \& Merikangas, K. The future of genetic studies of complex human diseases. Science 273, 1516-1517 (1996).

4. Manolio, T. A. et al. Finding the missing heritability of complex diseases. Nature 461, 747-753 (2009).

5. Dekker, J. M. et al. Heart rate-corrected QT interval prolongation predicts risk of coronary heart disease in black and white middleaged men and women: the ARIC study. J. Am. Coll. Cardiol. 43, 565-571 (2004).

6. Newton-Cheh, C. et al. QT interval is a heritable quantitative trait with evidence of linkage to chromosome 3 in a genome-wide linkage analysis: The Framingham Heart Study. Heart Rhythm 2, 277-284 (2005).

7. Priori, S. G. \& Napolitano, C. Genetics of cardiac arrhythmias and sudden cardiac death. Ann. N. Y. Acad. Sci. 1015, 96-110 (2004).

8. Arking, D. E. et al. Genetic association study of QT interval highlights role for calcium signaling pathways in myocardial repolarization. Nat. Genet. 46, 826-836 (2014).

9. Arking, D. E. et al. A common genetic variant in the NOS1 regulator NOS1AP modulates cardiac repolarization. Nat. Genet. 38, 644-651 (2006).

10. Newton-Cheh, C. et al. Common variants at ten loci influence QT interval duration in the QTGEN Study. Nat. Genet. 41, 399-406 (2009).

11. Pfeufer, A. et al. Common variants at ten loci modulate the QT interval duration in the QTSCD Study. Nat. Genet. 41, 407-414 (2009).

12. Kapoor, A. et al. An enhancer polymorphism at the cardiomyocyte intercalated disc protein NOS1AP locus is a major regulator of the QT interval. Am. J. Hum. Genet. 94, 854-869 (2014).

13. The ARIC Investigators. The Atherosclerosis Risk in Communities (ARIC) Study: design and objectives. Am. J. Epidemiol. 129, 687-702 (1989)

14. Rautaharju, P. M., Park, L. P., Chaitman, B. R., Rautaharju, F. \& Zhang, Z. M. The Novacode criteria for classification of ECG abnormalities and their clinically significant progression and regression. J. Electrocardiol. 31, 157-187 (1998).

15. Psaty, B. M. et al. Cohorts for Heart and Aging Research in Genomic Epidemiology (CHARGE) Consortium: Design of prospective meta-analyses of genome-wide association studies from 5 cohorts. Circ. Cardiovasc. Genet. 2, 73-80 (2009). 
16. Lupski, J. R. et al. Exome sequencing resolves apparent incidental findings and reveals further complexity of SH3TC2 variant alleles causing Charcot-Marie-Tooth neuropathy. Genome Med. 5, 57 (2013).

17. http://www.ncbi.nlm.nih.gov/SNP/ Database of Single Nucleotide Polymorphisms. Date of access:05/20/2016.

18. Pruitt, K. D. et al. RefSeq: an update on mammalian reference sequences. Nucleic Acids Res. 42, D756-63 (2014).

19. http://www.ncbi.nlm.nih.gov/geo/query/acc.cgi?acc=GSE16256 USCD Human Reference Epigenome Mapping Project. Date of access:01/01/2015.

20. http://annovar.openbioinformatics.org/en/latest/ ANNOVAR. Date of access:01/01/2015.

21. Wang, K., Li, M. \& Hakonarson, H. ANNOVAR: functional annotation of genetic variants from high-throughput sequencing data. Nucleic Acids Res. 38, e164 (2010).

22. Pollard, K. S., Hubisz, M. J., Rosenbloom, K. R. \& Siepel, A. Detection of nonneutral substitution rates on mammalian phylogenies. Genome Res. 20, 110-121 (2010).

23. Karolchik, D. et al. The UCSC Table Browser data retrieval tool. Nucleic Acids Res. 32, D493-6 (2004).

24. https://genome.ucsc.edu/cgi-bin/hgTables UCSC Table Browser tool. Date of access:01/01/2015.

25. http://www.hprd.org/ Human Protein Reference Database. Date of access:01/01/2015.

26. Keshava Prasad, T. S. et al. Human Protein Reference Database-2009 update. Nucleic Acids Res. 37, D767-72 (2009).

27. Roberts, A. M. et al. Integrated allelic, transcriptional, and phenomic dissection of the cardiac effects of titin truncations in health and disease. Sci. Transl. Med. 7, 270ra6 (2015).

28. Ng, P. C. \& Henikoff, S. Predicting Deleterious Amino Acid Substitutions. Genome Research 11, 863-874 (2001).

29. Adzhubei, I. A. et al. A method and server for predicting damaging missense mutations. Nat. Methods 7, 248-249 (2010).

30. http://exac.broadinstitute.org/ ExAC Browser. Date of access:01/01/2015.

31. Bazett, H. C. An analysis of the time-relations of electrocardiograms. Annals of Noninvasive Electrocardiology 2, 177-194 (1997).

32. Reardon, M. \& Malik, M. QT interval change with age in an overtly healthy older population. Clin. Cardiol. 19, 949-952 (1996).

33. Yang, H. et al. Sex differences in the rate of cardiac repolarization. J. Electrocardiol. 27, 72-73 (1994).

34. http://pngu.mgh.harvard.edu/ purcell/plink/ PLINK. Date of access:01/01/2015.

35. Purcell, S. et al. PLINK: a tool set for whole-genome association and population-based linkage analyses. Am. J. Hum. Genet. 81, 559-575 (2007)

36. Benjamini, Y. \& Hochberg, Y. Controlling the False Discovery Rate: A Practical and Powerful Approach to Multiple Testing. Journal of the Royal Statistical Society. Series B (Methodological) 57, 289-300 (1995).

37. Price, A. L. et al. Principal components analysis corrects for stratification in genome-wide association studies. Nat. Genet. 38, 904-909 (2006).

38. Taylor, M. et al. Genetic variation in titin in arrhythmogenic right ventricular cardiomyopathy-overlap syndromes. Circulation 124, 876-885 (2011).

39. Brun, F. et al. Titin and desmosomal genes in the natural history of arrhythmogenic right ventricular cardiomyopathy. J. Med. Genet. 51, 669-676 (2014)

40. Smith, E. \& Mani, A. Titin as a gene for conduction defects with and without cardiomyopathy (2160M). 64th Annual Meeting of The American Society of Human Genetics San Diego, California (October 20, 2014).

41. Leinonen, J. T. et al. Search for novel mutations predisposing to ventricular fibrillation without overt cause (614T). 65th Annual Meeting of The American Society of Human Genetics Baltimore, Maryland (October 8, 2015).

\section{Acknowledgements}

This work was supported by the U.S. National Institute of Health grants RO1GM104469 and RO1HL128782. The ARIC Study is carried out as a collaborative study supported by National Heart, Lung, and Blood Institute (NHLBI) contracts (HHSN268201100005C, HHSN268201100006C, HHSN268201100007C, HHSN268201100008C, HHSN268201100009C, HHSN268201100010C, HHSN268201100011C, and HHSN268201100012C), R01HL087641, R01HL59367 and R01HL086694. The authors thank the staff and participants of the ARIC study for their important contributions. Funding support for "Building on GWAS for NHLBI-diseases: the U.S. CHARGE consortium" was provided by the NIH through the American Recovery and Reinvestment Act of 2009 (ARRA) (5RC2HL102419). Data for "Building on GWAS for NHLBI-diseases: the U.S. CHARGE consortium" was provided by Eric Boerwinkle on behalf of the Atherosclerosis Risk in Communities (ARIC) Study, L. Adrienne Cupples, principal investigator for the Framingham Heart Study, and Bruce Psaty, principal investigator for the Cardiovascular Health Study. Sequencing was carried out at the Baylor College of Medicine Human Genome Sequencing Center (U54 HG003273).

\section{Author Contributions}

A.K. and A.C. designed the study; A.C., E.B., M.L.G. and D.E.A. contributed towards data generation; A.K., K.B., L.X., P.N., D.L., D.E.A. and A.C. performed data analysis; A.K., K.B. and A.C. wrote the manuscript; all authors reviewed the manuscript.

\section{Additional Information}

Supplementary information accompanies this paper at http://www.nature.com/srep

Competing financial interests: A.C. is on the Scientific Advisory Board of Biogen Idec and this potential competing interest is managed by the policies of the Johns Hopkins University, School of Medicine. All other authors have no competing financial interests to declare.

How to cite this article: Kapoor, A. et al. Rare coding TTN variants are associated with electrocardiographic QT interval in the general population. Sci. Rep. 6, 28356; doi: 10.1038/srep28356 (2016).

This work is licensed under a Creative Commons Attribution 4.0 International License. The images or other third party material in this article are included in the article's Creative Commons license, unless indicated otherwise in the credit line; if the material is not included under the Creative Commons license, users will need to obtain permission from the license holder to reproduce the material. To view a copy of this license, visit http://creativecommons.org/licenses/by/4.0/ 